\title{
Clinical-radiological relationship in fasciolosis: A case report
}

\begin{abstract}
Introduction: The Fasciolosis is a parasitosis. In most cases in human, the diagnosis is difficult for nonspecific symptoms parasite eggs and disposal flashing with stool. Finding Imaging, alterations biochemical and especially the serology contribute to the diagnosis in both acute and chronic stage Fever long, pain right upper quadrant and eosonophilia persistent should make suspect this disease.
\end{abstract}

Objective: To show the utility Diagnose of test image (Ultrasound and Computed Tomography) and the main findings in relation to the stage of the disease.

Case report: It is presented to clinical case of a patient with the diagnosis of liver Fasciolosis. In this particular case all tests were negative or non-specific being tests and the strong clinic led to the final diagnosis. The patient was referred to IPK and was treated with a single dose of triclabendazole, with excellent clinical response.

Conclusion: Clinic suspicion of Fasciolosis next to imaging studying can contribute to the diagnosis of this disease. It is necessary for clinical and medical radiologist to include the Fasciolosis in the differential diagnosis of focal liver injuries especially in the context of a eosonophilia.

Keywords: fasciolosis, liver fasciola, ultrasound Computed tomography, imaging diagnosis radiology, focal liver injuries
Volume 6 Issue 2 - 2018

\author{
Aliet Arrue Guerrero, Jania Acosta Lopez, \\ Yasmel Tarafa Rosales \\ Specialist Degree in Imaging, Spain
}

Correspondence: Aliet Arrue Guerrero, Specialist Degree in Imaging, Bachelor of Imaging, Instructor, Spain, Email aaguerrero@infomed.sld.cu

Received: March 12, 2018 | Published: April 03, 2018

\section{Introduction}

Fasciolosis is a zoonosis caused by the entire Fasciola hepatica trema, which affects herbivorous vertebrate animals (cows, sheep, and goats, among others) and humans. The infection is acquired due to the intake of various raw aquatic plants, some terrestrial, or water contaminated with metacercariae, the infecting form. It is estimated that there are at least 2.4 million people infected in 70 countries. No continent is free of species of the genus Fasciola (Fasciola hepatica and F. gigantica), and it is considered that where there are cases of these parasites in animals, there are also human cases. ${ }^{1,2}$

The diagnosis is based on the epidemiological antecedents, symptoms and clinical signs; The specific diagnosis is the finding of their eggs in faeces or biliary drainage, also in the detection of anti- bodies and antigens. ${ }^{3,4}$ In Cuba, bovine Fasciolosis is enzootic throughout the country. In humans it has been presented in the form of outbreaks that have involved hundreds of people and some sporadic cases, existing in today 1 to underreporting of Fasciolosis not be a notifiable disease to the health system. ${ }^{5}$

Radiology plays a fundamental role in the diagnosis, evolution and follow-up; Imaging modalities include Ultrasound and Computed Axial Tomography. The present investigation presents a case of Fasciolosis with the objective of reviewing the imaging findings by ultrasound and by Tomography of Fasciolosis, showing its important clinical and imagenological relationship.

\section{Clinical case}

Male patient of 37years of age, white race, resident in Habana del Este municipality of Havana province, with previous health history that comes to consultation for presenting prolonged febrile syndrome with 56days of evolution, febrile peaks of 38-39 degrees, accompanied by chills and decay. Urinary sepsis was determined as initial diagnosis so antimicrobial treatment was imposed for 10 days. There is no improvement in the clinical picture and the febrile symptoms persist. The patient is admitted to the hospital service of the Central Hospital in Havana. On physical examination, painful abdomen on palpation in the right hypochondrium and epigastrium without other alterations Laboratory tests are performed or presenting Haemoglobin: 12gl, Leucogram which yields an Eosonophilia of 66\% and an Erythrosedimentation: 67. Serial blood cultures and urine cultures they were negative. Likewise, the serology was negative. Within imaging studies abdominal ultrasound reporting hepatomegaly that exceeds be $2 \mathrm{~cm}$ costal margin with the presence of a hypo echoic image with nodular, peripheral tendency without flow Doppler level right lobe study (Figure 1) interpreting c indicated omo pyogenic liver abscess therefore imposes treatment with antimicrobial therapy (Metronidazol EV) (Figure 1). Ultrasound: nodular, hypoechoechoic, peripheral, non-vascularised image at the level of the right lobe of the liver clinically the patient improved his general condition, but he kept the febrile syndrome accompanied by diffuse abdominal pain. By keeping the same ultrasound image last month of treatment study it was conducted by $\mathrm{T}$ omografía who reported abdominal the presence of density areas of irregular appearance, in the right lobe of the liver in segments VII and VIII and endovenous study showed lesions with poor contrast capitation both Portal and late phase (Figure 2). Computed tomography with intravenous contrast: hypo dense areas of irregular appearance at the level of segments VII and VIII of the liver with poor contrast uptake Ultrasound images suggested the diagnosis of parasitosis Fasciola hepatica but also by positron raised the differential diagnosis of Focal nodular hyperplasia at que sugi river echo directed FNA that reported cytological Negative of 
neoplastic cells and inflammatory infiltrate with abundant eosinophils Given this possible diagnosis, endoscopy was performed, which reported alkaline pangastropathy with a duodenal swab for negative parasitological study. Conical crown was made on several occasions resulting negative and biliary drainage was indicated to be not useful for diagnosis. In view of Clinical and Imaging suspicion of Fasciola hepatica, it was decided to perform a weekly evaluative ultrasound for 4 more weeks, observing at the end of the fourth week echogenic

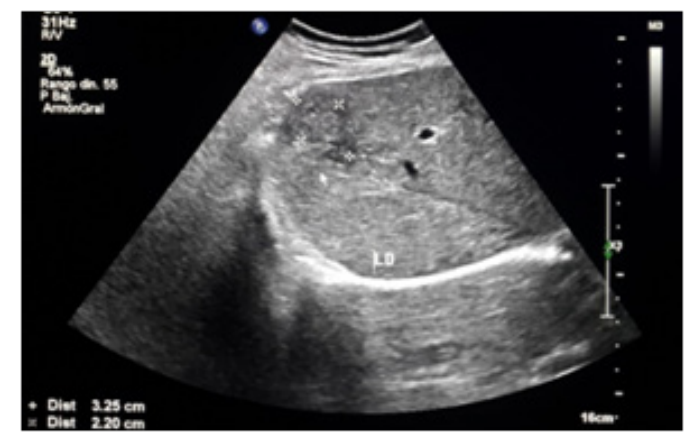

Figure I Ultrasound.

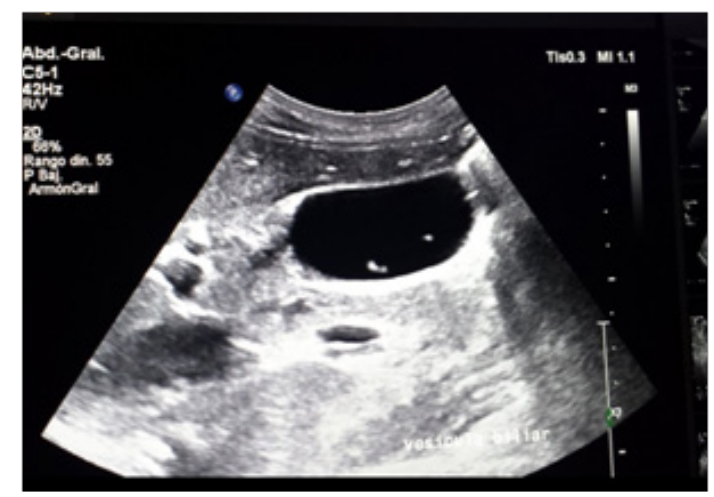

Figure 3 Evolutionary ultrasound.

\section{Discussion}

The biological cycle of Fasciola hepatica ( liver fluke) requires 2hosts: the herbivorous animals (cattle, sheep, goats, horses, rabbits, hares, deer, others) and the human, all of them definitive hosts and the freshwater lung snails of the genus Lymnaea spp. as intermediary hosts. ${ }^{1,2}$

The human is infected by ingesting aquatic plants (including watercress, lettuce), other short-stemmed plants, and terrestrial, grown in the vicinity of freshwater bodies contaminated with metacercariae. The infection can also be acquired through the ingestion of contaminated water., ${ }^{4,6}$ The de-escalation of metacercariae occurs in the small intestine, thanks to components of bile. The juvenile forms cross the intestinal wall, migrate through the peritoneal cavity, penetrate the hepatic parenchyma, where they have a growth phase that lasts about 2 months and end their development in the bile ducts, adult habitat. They can survive in the host for 9-13.5years. . $^{5,7}$

\section{Epidemiology}

The impact of Fasciola hepatica and Fasciola gigantica infections images, elongated with movements inside the gallbladder and an echogenic image without acoustic shadow in the canal. Coledochus suggesting the diagnostic imaging of adult Fasciola parasites in the bile ducts (Figure 3). Evolutionary ultrasound: echogenic images, free, with movement at the level of the gallbladder. The patient was referred to the Institute of Tropical Medicine (IPK) where the diagnosis was verified, imposing treatment with a single oral dose of Triclabendazole with excellent therapeutic response.

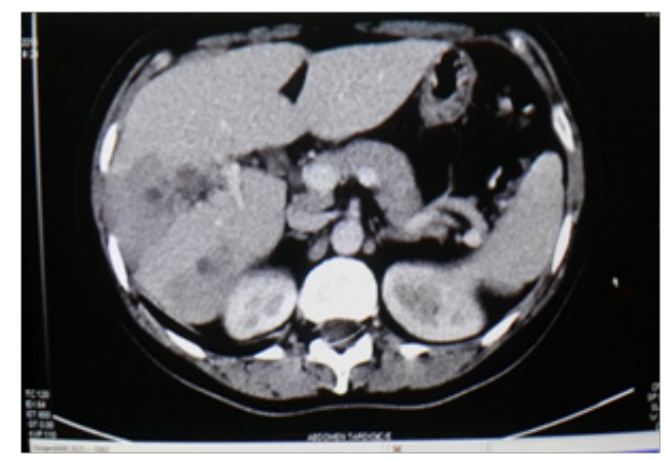

Figure 2 Computed tomography.

has been underestimated in relation to its prevalence in humans and economic losses in livestock. The association of the intake of raw vegetables has been identified. Watercress and radish are considered the most risky, as are lettuce, corn cob and spinach. ${ }^{5,6}$ In Cuba fascioliasis is a disease of the livestock and is responsible for significant losses in the livestock and food industries, which allows it to be considered as the zoonotic disease of major economic importance and of great interest to veterinarians, which mainly affects cattle and sheep in both state and private sectors. ${ }^{5}$ The first autochthonous case of human Fasciolosis in Cuba was reported in 1931, at the Sanatorium La Covadonga, Province of Havana, by Doctors Kouri and Arenas; subsequently, another six cases were registered in only 8 months. From then until 1944, more than 100 sporadic cases were diagnosed in Cuba, representing more than a third of the total sporadic cases recorded in the world medical literature of that time. At the end of 1944 more than 50 cases of Fasciolosis is reported in epidemic form in Pinar del Río and in 1948 more than 100 cases were also reported in the same province. ${ }^{8}$ Other outbreaks have occurred in the province of Pinar del Río, as for example in 1995 when an outbreak of 82 cases was reported in La Palma, and in 2003 in Consolation del Sur. Other provinces have also been affected by the occurrence of epidemic outbreaks, such as Villa Clara and Sancti Spíritus in 1983, and in 1996 an outbreak of 390 cases was reported in Esmeralda, Camagüey province. ${ }^{8}$ Currently, sporadic human cases continue to appear. The presence of intermediate hosts, as well as the habit of eating vegetables such as cress and lettuce poorly washed, serve as transport to the agent and provide the necessary conditions for the maintenance of the infection. ${ }^{5,8}$

\section{Clinical spectrum}

The acute Fasciolosis syndrome usually lasts three to four months; It is characterized by fever of variable intensity, severe pain of the right hypochondrium or epigastric pain, myalgias, arthralgias, headache, nausea, Eosonophilia $>5000 / \mathrm{mL} 3$ and hepatomegaly (migratory phase of hepatic invasion). ${ }^{3,4}$ The clinical spectrum has been divided into 2 stages: an acute or invasive phase that responds to 
the migration of immature flukes from the intestine to the bile ducts. During the invasive period, the clinical picture includes: localized pain in the epigastrium and / or upper right quadrant with irradiation to the scapula on the same side, hepatomegaly, irregular febrile outbreaks, nausea, diarrhoea, malaise, myalgias, arthralgias, urticaria fleeting with dermographism infrequent. In blood count, leukocytosis can be seen with deviation to the left, anemia and hypereosinophilia $(30-70 \%){ }^{8,9}$ In this patient, only moderate Eosonophilia could be observed from the beginning of the clinical picture. In this phase, ELISA and indirect immunofluorescence tests can be performed, and efforts have been concentrated on obtaining excretion /secretion (E/S) antigens and recombinant molecules to improve serological tests, which are very useful in the early diagnosis of disease ${ }^{5,6,9}$ The chronic phase (or state): It occurs after about 3-5 months post-infection, and the clinical manifestations are associated with the presence of Fasciola $\mathrm{s}$ in the bile ducts. It is characterized by signs and symptoms related to biliary obstruction (partial or complete in more severe cases) and the degree of inflammation: abdominal pain, nausea, vomiting, anorexia, soft hepatomegaly, fever, a picture similar to a chronic exacerbated cholecystitis. Consequences of the chronic presence of parasites are considered: cholecystitis, cholangitis, bacterobilia, pancreatitis, per portal cirrhosis, and liver fibrosis. It has not yet been associated with the development of cholangiocarcinoma. Jaundice becomes evident in the presence of complete obstruction, which requires surgery or emergency endoscopy. ${ }^{10,11}$ Cases have been reported with significant parasitic load and absence of clinical manifestations, which can constitute a silent threat, since the parasites can survive several years, and if the patient is asymptomatic or with nonspecific clinical manifestations, the liver damage could be irreversible. $5,11,12$ In this phase, parasitoscopic examinations are positive after 3-4months after infection, when adult parasites eliminate eggs and these can be identified in coproparasitoscopic tests. Concentration by sedimentation and protection of coproantigens. ${ }^{2,8}$ You can also make study of 1 duodenal content and tissue biopsy. Endoscopic studies may be necessary to identify filling defects in bile ducts or vesicles, and to collect juvenile or adult forms of the parasite. ${ }^{8,9}$ In the case reported all these last diagnostic methods were negative, maintaining only the Eosonophilia, using imaging tests for the definitive diagnosis of it.

\section{Imagenological appearances}

The contribution of the imaging tests for the diagnosis will depend on the disease phase in which the patient is located, comprising from the image point of view two entities that are the liver form (coinciding with the acute clinical phase) and the biliary form (corresponding to the chronic phase and $\mathrm{n}$ fermedad) ${ }^{5,13}$ In Fasciolosis hepatica, its appearance require a high degree of suspicion since it can be indistinguishable from other focal liver lesions.

\section{Ultrasound}

In hepatic fascioliasis, hypo echoic, anfractuous, well-defined focal lesions of variable size can be observed, with a tendency to accentuate in $96 \%$ of cases (similar to the case reported), although variable echo genicity is also described. However, it is not always possible to visualize the paths that give it the characteristic stamp, so its role in acute Fasciolosis is less. The presence of per portal adenopathies is also described in $74 \%$ of cases, although this finding is nonspecific and is present in acute and chronic hepatitis, abdominal neoplasm's, abdominal tuberculosis and sclerosing cholangitis, among other pathologies. ${ }^{13,14}$ In Biliary Fascioliasis, the role of ultrasound is greater, given that it is able to confirm parietal thickening of the common bile duct with moderate dilation, although this finding is also seen in pathologies such as sclerosing cholangitis and AIDS cholangitis . More specific is the observation of mobile structures in the gallbladder and / or common bile duct, representing the adult form of Fasciola, ${ }^{14,15}$ a finding found in the reference patient.

\section{Computed tomography}

In Fasciolosis hepatica, it can present as nodular or tortuous lesions, with enhancement after variable contrast, although in $80 \%$ it allows its better delimitation in late phases. However, the most characteristic of these lesions is their sub capsular location, with tortuous paths that adopt a centripetal direction in the direction of intrahepatic bile ducts, whose appearance makes some authors propose it as highly suggestive of Fasciolosis (Figure 2). ${ }^{16-18}$ Hepatic capsule enhancement with contrast injection is also described, which correlates with thickening and inflammation of the capsule observed in laparoscopy. Another element to consider is the sub capsular hematoma, an infrequent complication, but one that undoubtedly requires imaging control. In relation to biliary fasciolosis, the role of $\mathrm{CT}$ in its diagnosis is smaller, being able to only show minimal dilation of the extra hepatic bile duct, being a non-specific finding. ${ }^{17}$ In the patient reason for discussion, both Tomography and Ecography could be useful, the latter being the one that contributed most to the definitive diagnosis, coinciding with the findings reported in the literature according to the clinical phase of the disease.

\section{Conclusion}

The clinical suspicion of Fasciolosis with imaging studies may contribute to diagnosis n, 1 showing exact stage of the disease. It is necessary that the clinician and the radiologist in particular, include Fasciolosis in the differential diagnosis of focal liver lesions, especially in the clinical context of an Eosonophilia.

\section{Acknowledgements}

None.

\section{Conflicts of interest}

The authors deny conflicts of interest in relation to the research presented.

\section{References}

1. Carrada Bravo T. Fasciolosis. Rev Mex Patol Clin. 2005;52(2):83-96.

2. Martinez Sanchez R, Domenech Canete I, Millan Marcelo JC, et al Fascioliasis, clinical epidemiological review and diagnosis. Rev Cuban Hig Epidemiol. 2012;50(1).

3. More CS, Valero MA, Bargues MD. Chapter 5. Fascioliasis. Adv Exp Med Biol. 2014;(766):77-114.

4. Lazo Molina L, Garrido Acedo R, Cardenas Ramirez B, et al. Endoscopic extraction by ERCP of Fasciola hepatica viva: report of two cases and review of the literature. Rev Gatroenterol Peru. 2013;33(1):75-81.

5. Domenech Canete I. Hepatic abscess, a rare form of presentation of Fasciolosis in Cuba. Rev P anam Infectol. 2011;13(2):53-56.

6. Tolan Robert W. Fascioliasis Due to Fasciola hepatica and Fasciola gigantica Infection: An Update on This 'Neglected' Neglected Tro pical Disease. LabMedicine. 2013;42(2):107-116.

7. Zumaquero Rios JL, Sarracent Perez J, Rojas Garcia R, et al. Fascioliasis 
and Intestinal Parasitoses Affecting Schoolchildren in Atlixco, Puebla State, Mexico: Epidemiology and Treatment with Nitazoxanide. PLoS Negl Trop Dis. 2013;7(11):e2553.

8. Martinez SR, Domenech CI, Millan MJ, et al. Fascioliasis, clinical epidemiological review and diagnosis. Cuban Journal of Hygiene and Epidemiology. 2012;50(1):1561-3003.

9. Lopez PA, Silva FC, Busel. Fasciolasis hepatica: Report of a case and review of the literature. Rev Chil Radiol. 2004;10(3):118-123.

10. Martinez LR, Ruiz TJ, Diaz CF, et al. Diagnosis of biliary tract fasciolosis by imaging. Rev cubana med trop. 2000;52(2):145-147.

11. Eleven Diaz R. Human Fasciolosis, 10 years of experience in the Institute of Tropical Medicine "Pedro Kourí”. Master's Thesis: USA; 2008.

12. Cabada MM, Lopez M, Cruz M, et al. Treatment Failure after Multiple Courses of Triclabendazole among Patients with Fascioliasis in Cusco, Peru: A Case Series. PLoS Negl Trop Dis. 2016;10(1):e0004361.
13. Quintana RY, Lopez BC, Perez CL, et al. Imaging diagnosis of Fasciola hepatica infection. Els evier Radiology. 2014;56:284

14. Corti ME, Palmieri OJ, Mendez N, et al. Diagnosis by imaging in hepatic fascioliasis. Revista Argentina de Radiologia. 2006;70(3):197-200.

15. Yesildag A, Yildiz H, Demirci M, et al. Biliary fascioliasis: Sonographic appearance patterns. Journal of Clinical Ultrasound. 2009;37(1):26-30.

16. Cosme A, Ojeda E, Poch M, et al. Sonographic findings of hepatic lesions in human fascioliasis. J Clin Ultrasound. 2003;3(7):358-363.

17. Patel NU, Bang TJ, Dodd GD. CT findings of human Fasciola hepatica infection: case reports and review of the literature. Clin Imaging. 2016;40(2):251-255.

18. Sezgi C. Pulmonary findings in patients with fascioliasis. Mediterranean Medico Act. 2013;29:841-845. 